\title{
A Feature-Based Environment for Digital Games
}

\author{
Victor T. Sarinho ${ }^{1}$, Antônio L. Apolinário Jr. ${ }^{2}$, and Eduardo S. Almeida ${ }^{3}$ \\ ${ }^{1}$ DEXA - State University of Feira de Santana (UEFS), \\ Feira de Santana, Bahia, Brazil \\ vsarinho@gmail.com \\ ${ }^{2}$ Federal University of Bahia (UFBA), \\ Salvador, Bahia, Brazil \\ ${ }^{3}$ Reuse in Software Engineering (RiSE), \\ Salvador, Bahia, Brazil \\ \{apolinario, esa\} adcc.ufba.br
}

\begin{abstract}
Digital games can be considered as an important software development area in our society. This paper proposes the Object Oriented Feature Modeling (OOFM) usage in the digital game domain. It aims to represent and manipulate distinct game features, defined by NESI and GDS models, in a parameterized and hierarchical way. As a result, a Feature-based Environment for Digital Games (FEnDiGa) is provided, a product line platform able to integrate and adapt represented game features in different types of available game engines.
\end{abstract}

Keywords: Digital game domain, game features, OOFM, FEnDiGa.

\section{Introduction}

Digital games can be considered as an important software development area in our society. Analyzing the digital game domain, it is possible to verify multiple examples of game genres and categories presenting common features among them [1], distinct modeling approaches able to represent digital games [2, 3], and a great influence of game engines during game design and implementation activities [4].

This paper proposes the Object Oriented Feature Modeling (OOFM) [5] usage in the digital games domain. It aims to manage distinct game features provided by NESI [2] and GDS [3] models in a specialized OOFM structure, providing the game logic, rules and goals (the G-Factor [4]) in a parameterized and hierarchical way. As a result, the Feature-based Environment for Digital Games (FEnDiGa) is provided, a software structure able to integrate and adapt represented game features in different types of available game engines.

The reminder of this paper presents three game development steps necessary to provide and use the FEnDiGa structure: 1) the variability documentation of digital games in a generic way; 2) the creation of a feature-based environment and process able to work with generic game features; and 3) the configuration of feature game resources in order to provide a final game. 


\section{Step 1: Documenting the Game Variability}

An interesting problem in the digital game domain is the representation of game characteristics. There are many types of game representation approaches in the literature presenting a lot of game elements, game behaviors and so on. In a previous work [2, 3], two distinct generic feature models were proposed (Fig. 1): the NESI model, documenting the conceptual variability of a digital game, and the GDS model, documenting the implementation variability of a digital game.

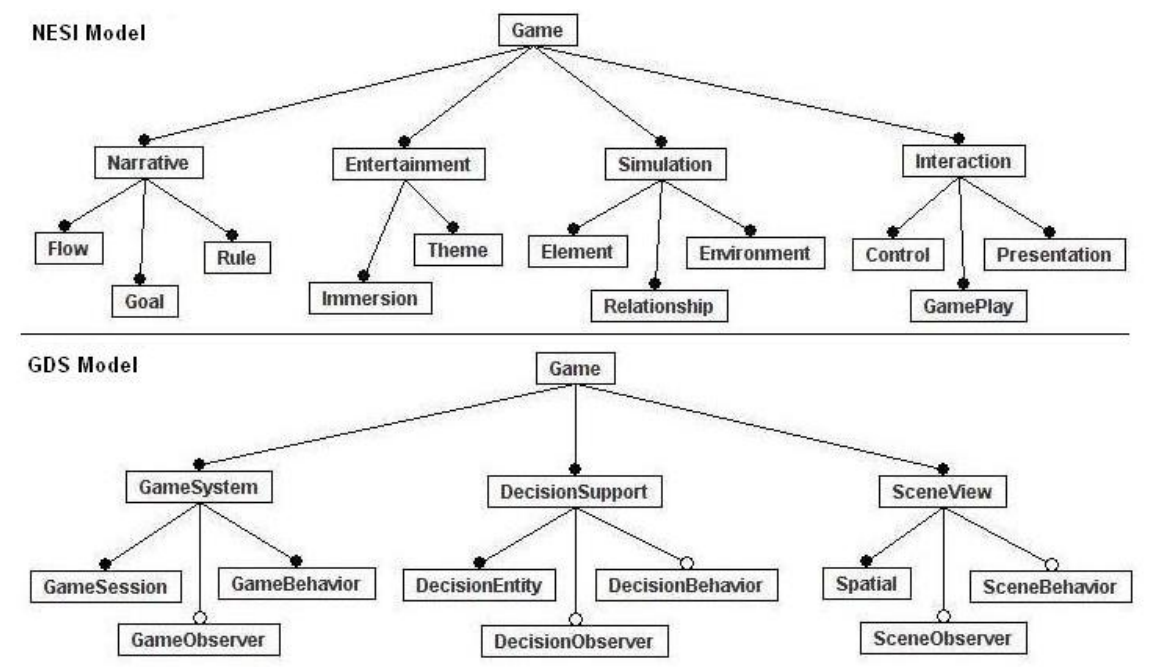

Fig. 1. The NESI and GDS models

The NESI model [2] defines a digital game as a combination of Narrative, Entertainment, Simulation and Interaction. A Narrative is a Flow, a dynamic script trying to achieve Goals following defined Rules of a game. Entertainment is represented by the player Immersion during the gameplay, following a Theme proposal to integrate the player and the game in the proposed reality. Simulation is a combination of Elements (resources to play) and respective Relationships that happen in a defined Environment (spaces to play). The human Interaction is represented by Control (game inputs) and Presentation (game outputs), consolidated by the GamePlay that represents a current game execution as a whole. With these main features, other subfeatures related to game concepts were defined, providing more than 350 conceptual game features to be configured and manipulated by game designers.

On the other hand, the GDS model [3] defines a digital game as a combination of GameSystem, DecisionSupport and SceneView main features, generating more than 250 related sub-features. The GameSystem feature is the main joint point of a game. It is responsible to control the game execution, describing available GameBehaviors, GameContext and GameObservers for a digital game. The DecisionSupport feature is an effort to integrate some artificial intelligence strategies used by different digital 
games, presenting DecisionEntities, the ContextState of each DecisionEntity, DecisionObservers monitoring DecisionEntities values, and predefined DecisionBehaviors able to read and update DecisionEntities and ContextStates values during their executions. The SceneView feature is a collection of SceneNodes distributed by Spatial sessions with various SceneBehaviors and SceneObservers able to perform scene actions in a game. A SceneNode represents a collection of information about the scene, describing specific Location and BoudingVolume for collision detection among other scene nodes, as well as hierarchical information about AudioNode, GraphicNode and PhysicsNode simultaneously.

\section{$3 \quad$ Step 2: Creating a Feature-Based Environment}

NESI and GDS models describe generic and distinct Feature Models (FMs) and Feature Configurations (FC) for digital games. OOFM provides basic elements (FeatureType, Feature, and so on) to work with represented FMs and FCs. The OOFM Framework [5] is a combination of OOFM elements plus extra classes, interfaces and packages that follows the Model-View-Controller (MVC) architectural pattern [6]. It defines abstract FeatureState, feature observer, FeatureBehavior and Adapter structures in order to organize the production of final system for represented FMs and FCs.

FEnDiGa is a concrete specialization of the OOFM Framework focused on the digital game domain (Fig. 2). It is a game development environment that: represents game features based on NESI and GDS models via OOFM resources; combines game features using the proposed OOFM Framework structures, and implements game features using specialized adapters of desired game engines.

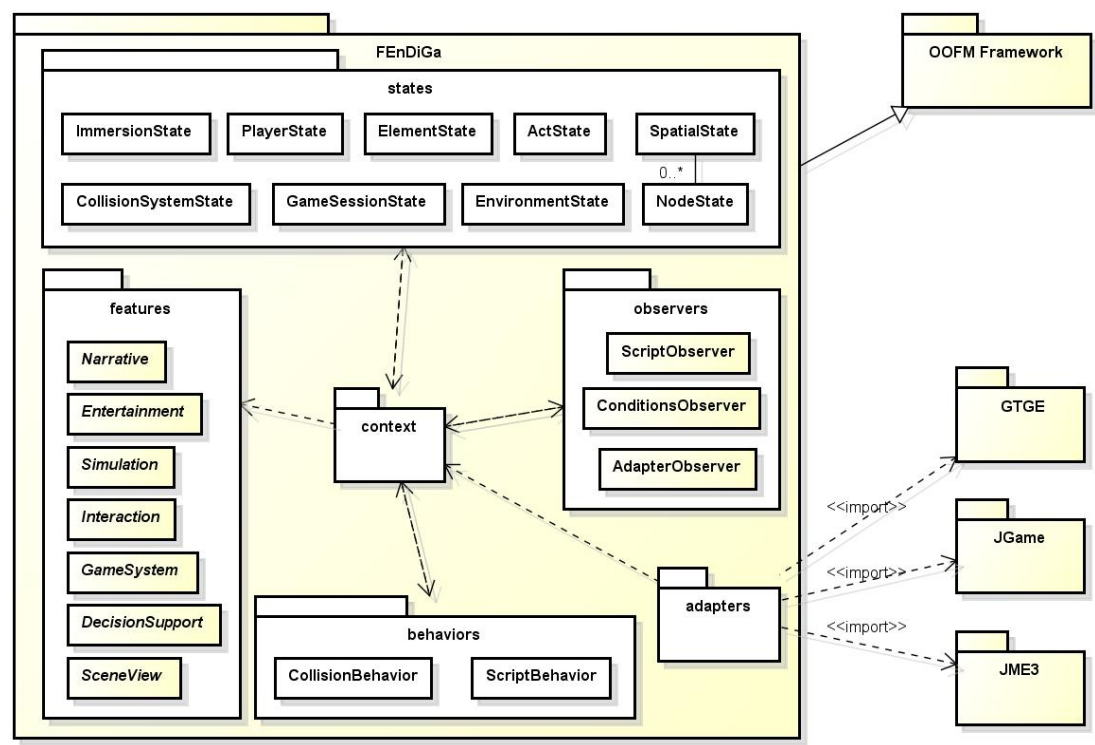

Fig. 2. The FEnDiGa architecture 
The representation of game features are performed by FeatureClass structures [5]. They are created for each main feature of the respective NESI and GDS models (game feature containers), presenting feature methods that prepare each featureModel and respective feature Configurations for games.

The integration of represented game features can be performed by FeatureState, feature observer and FeatureBehavior specializations. Using a FeatureState, different game features (but representing similar characteristics) can be used to model a common state. A good example is the ElementState class, which is a combination of Element, Avatar and DecisionEntity game features.

FeatureState instances can be monitored by FeatureStateObserver instances, which are triggered whenever a FeatureState update is applied. They verify collisions among Element instances or updates in current values of Avatar Skills, for example. When a feature observer is triggered, a FeatureBehavior instance is performed, providing support for game behaviors described by game features, such as Action, Relationship, GameBehavior, DecisionBehavior and SceneBehavior.

Finally, for the implementation of game features, it consists of preparing specific Adapter instances to reflect available features and FeatureState instances in the desired game engine. They are the main interface among game engines procedures and represented game features in order to execute the configured game.

Moreover, considering the FEnDiGa development steps, features and states can be configured by Game Designers and Game Programmers, observers and behaviors can be contextualized by Game Programmers, and adapters can be provided and used by Game Engine Specialists and Game Programmers, respectively. Fig. 3 illustrates this game development process, describing their game actors and important game development activities.

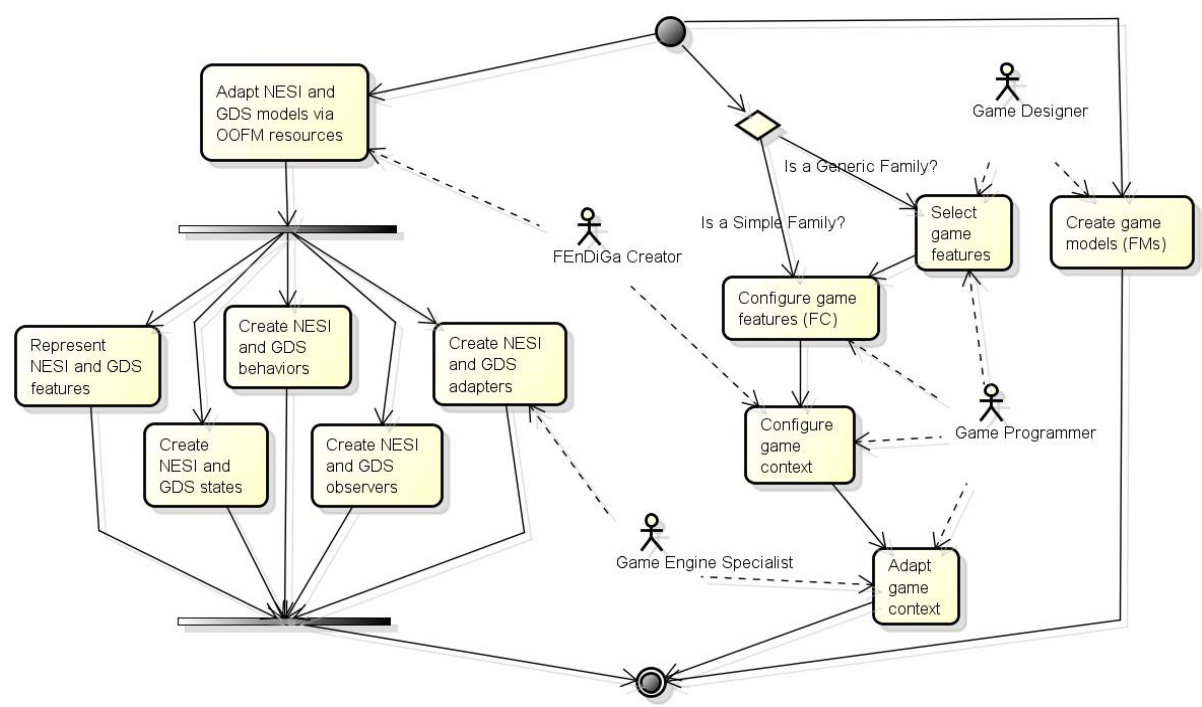

Fig. 3. The FEnDiGa development process 


\section{Step 3: Configuring the SimplifiedPacman Game}

With FEnDiGa structures ready to be instantiated by the FEnDiGa development process, the next step is the development of a final game (SimplifiedPacman in this case) by the configuration and interpretation of adapted game features.

Initially, identified game features based on NESI and GDS models are instantiated via FEnDiGa by the game developer. Next, several game states, such as pacmanState and pillState, are created to represent and integrate instantiated features. Feature updates and the notification of FeatureState updates are performed by behavior instances, which are defined by ScriptBehavior (such as pacmanDieBehavior) and FeatureBehavior specializations (movePacmanBehavior, for example). Also, for feature observer instances based on condition evaluation (collisionSystemObserver) and defined ScriptObserver (lifeEndEvent) results, they evaluate desired game states and indicate which behaviors must be performed, according to observer results. Finally, for the adaptation process, Adapter interfaces are implemented, such as SimplifiedPacmanSpriteAdapter and SimplifiedPacmanJGObjectAdapter, in order to adapt available engine resources (such as Sprite and JGObject) for defined FEnDiGa structures. They are used by engine procedures (such as init, start, render, and so on) in order to associate an engine behavior for each adapted state in a game.

As a result, by the proposed configuration of features, states, behaviors, observers and adapters, a complete structure for the SimplifiedPacman game, ready to be performed, is provided. Fig. 4 illustrates the SimplifiedPacman final architecture, adapted to three distinct game engines (GTGE, JGame and JME3) [7].

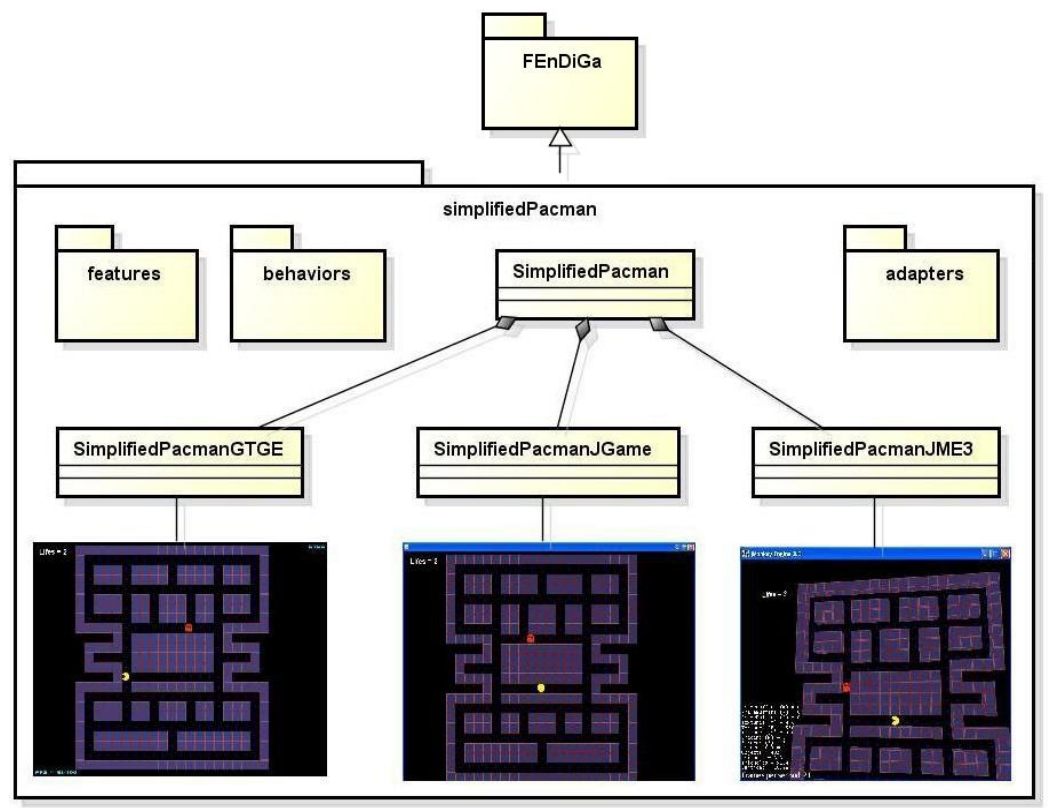

Fig. 4. The SimplifiedPacman game achitecture 


\section{Conclusions and Future Work}

This paper presented the application of the OOFM technique in the development of digital games represented by NESI and GDS feature models. For that, the FEnDiGa structure and development process were defined, providing a game development environment able to integrate conceptual and implementation aspects of games.

By the FEnDiGa usage, the game development work can be resumed to: instantiate representative features of a game; define states, observers and behaviors to represent a game context based on instantiated features; and define which states will be worked by adapters during the game execution in a final game engine.

Several types of combinations among defined FEnDiGa structures can also be performed, allowing as a result a great level of reuse for game categories represented by features (the game mixing). In addition, NESI and GDS specializations can be also supported, allowing the production of customized frameworks for games and game sub-domains (FEnDiGa-RPG) according to designer interests.

In other words, by the FEnDiGa usage, the game logic becomes standardized (based on NESI and GDS models), portable (G-Factor [4] in an independent way via Adapters usage) and reusable (distinct combination of game features) in a new software development approach.

For future work, some extra activities will be performed, such as the evaluation and simplification of NESI and GDS models, the development of FEnDiGa supporting tools, and the FEnDiGa usage in different game development approaches (engines, frameworks), categories (RPG, FPS, Adventure) and complexities (complete games instead of simplified versions, collaborative development, etc.).

\section{References}

1. Wolf, M.: The Medium of the Video Game. University of Texas Press (2002) ISBN: 029279150X

2. Sarinho, V., Apolinário, A.: A Feature Model Proposal for Computer Games Design. In: Proceedings of the VII Brazilian Symposium on Computer Games and Digital Entertainment, Belo Horizonte, pp. 54-63 (2008)

3. Sarinho, V., Apolinário, A.: A Generative Programming Approach for Game Development. In: Proceedings of the VIII Brazilian Symposium on Computer Games and Digital Entertainment, Rio de Janeiro, pp. 9-18 (2009)

4. Binsubaih, A., Maddock, S.: Game Portability Using a Service-Oriented Approach. IJCGT 2008, Article ID 378485, 7 pages (2008)

5. Sarinho, V., Apolinario, A.: Combining feature modeling and Object Oriented concepts to manage the software variability. In: Proceedings of the IEEE IRI 2010, August 4-6, pp. 344 349 (2010)

6. Krasner, E., Pope, T.: A cookbook for using the MVC user interface paradigm in Smalltalk80. J. Object Oriented Program 1, 26-49 (1988)

7. List of game engines. From Wikipedia, the free encyclopedia, http://en.wikipedia. org/wiki/List_of_game_engines 